\title{
El discurso del CEO: el pensamiento de los CEO respecto a la visión del futuro de la sociedad en el ámbito económico*
}

\author{
The CEO's discourse: The CEO's thought about a society's \\ economic vision of future
}

\section{O discurso do CEO: o pensamento dos CEOs em relação à visão do futuro da sociedade no âmbito económico}

Recibido: 4 de junio de 2019 Revisado: 15 de julio de 2019 Aceptado: 7 de agosto de 2019

Javier Muñoz Oroะco*

Universidad Vasco de Quiroga, Morelia, México

Cómo citar este artículo: Muñoz Orozco, J. (2019). El discurso del CEO: el pensamiento de los CEO respecto a la visión del futuro de la sociedad en el ámbito económico. Signos. Investigación en Sistemas de Gestión, 12(1),33-51. DOI: https://doi.org/10.15332/24631140.5419

\section{RESUMEN}

En el presente artículo se analizan las declaraciones de 24CEO de empresas multinacionales de las más importantes en ingresos y en tecnología, según el listado de la revista Fortune, en busca del pensamiento que subyace a su discurso acerca del futuro económico de las empresas en el contexto internacional. Se tomó como material de investigación el texto de sus entrevistas a medios especializados, sus declaraciones a diversos públicos y sus discursos oficiales en reuniones empresariales. Con la ayuda de un método cualitativo de división del texto en

\footnotetext{
Artículo de investigación.

** Doctor en Administración. Docente investigador. Universidad Vasco de Quiroga, Morelia, México. Correo electrónico jamunoro@hotmail.com Orcid: 0000-0002-5359-9761.
} 
unidades de pensamiento (Herzberg, 1959), y el empleo de comparaciones constantes con la visión de los especialistas en temas de futuro económico (Glaser y Strauss, 1967/2006), se obtuvieron seis temas centrales en donde aparece que en lo general los CEO están encaminando a sus organizaciones en el sentido que marcan los expertos en cuestiones de tecnología, medio ambiente, relaciones con los gobiernos y cambio en el modelo capitalista. Se hace mención de algunas excepciones. Los CEO advierten el paso de un cambio gradual en cuestiones tecnológicas, a una aceleración exponencial en las décadas a venir.

Palabras clave: CEO, metadiscurso, visión de futuro, innovación tecnológica, gestión empresarial.

\section{ABSTRACT}

In this article, the statements of 24 Chief Executive Officers (CEOs) of multinational companies of the most important in income and technology are analyzed, according to the Fortune magazine listing, in search of the thought that underlies their speech about the economic future of companies in the international context. The text of their interviews with specialized media, their statements to various audiences, and their official speeches at business meetings, were taken as research material. With the help of a qualitative method of dividing the text into units of thought (Herzberg, 1959), and the use of constant comparisons with the specialists' vision in topics of economic future (Glaser and Strauss, 1967/2006), six central themes were obtained where it is showed that $\mathrm{CEO}$ s are generally directing their organizations in the sense marked by experts in technology, environment, relations with governments, and change in the capitalist model. Mention is made of some exceptions. CEOs warn of a gradual change in technological issues, at an exponential acceleration in the decades to come.

Keywords: CEO, metadiscourse, future vision, technological innovation, business management.

\section{RESUMO}

No presente artículo são analisadas as afirmações de 24 CEOs de empresas multinacionais das mais importantes em renda e tecnologia, conforme a lista da revista Fortune, em busca do pensamento subjacente a seu discurso sobre o futuro económico das empresas no âmbito internacional. Foi considerado como material de pesquisa o texto de suas entrevistas a mídias especializadas, seus depoimentos perante diversos públicos e seus discursos oficiais em reuniões empresariais. com a ajuda de um método qualitativo de divisão do texto em unidades de pensamento (Herzberg, 1959), junto com o emprego de comparações com a visão dos especialistas em questões de futuro econômico (Glaser e Strauss, 1967/2006), surgiram seis tópicos centrais mostrando que em geral os CEOs estão encaminhando suas organizações na direção marcada pelos especialistas no que se refere a assuntos de tecnologia, meio ambiente, relações com os governos e mudança no modelo capitalista. São incluidas algumas resavas. Os CEOs destacam o passo de uma mudança gradativa em questões tecnológicas, para uma aceleração exponencial nas décadas vindouras.

Palavras-chave: CEO, metadiscurso, visão de futuro, inovação tecnológica, gestão empresarial. 


\section{INTRODUCCIÓN}

En una conferencia ante directores de empresas canadienses, Henry Mintzberg (2017) hizo ver la contradicción que existe entre algunas verdades proclamadas por la gestión empresarial y la realidad imperante en el mundo de las organizaciones. Una de esas verdades proclamadas se refiere al hecho de que el mundo de los negocios está en constante cambio, cuando en realidad los hechos muestran lo contrario.

Esta contradicción entre las verdades proclamadas y la realidad de los negocios da fe de la gran dificultad que tiene la sociedad para adaptarse a los cambios y vivir nuevas realidades. Sucede tanto en el mundo de los negocios como en la administración pública, y en las creencias de los seres humanos. En cualquier caso, para abrir camino hacia las nuevas realidades, hacia los hechos que de cualquier manera van a suceder, existen individuos con la capacidad para adelantarse y prever lo que depara el futuro, y que además cuentan con la capacidad y la autoridad para tomar decisiones siguiendo estas tendencias (Jaques y Cason, 2013).

En este sentido, Elliott Jaques (2000, 2006) y Jaques et al. (2013) nos ofrecen una herramienta de análisis que nos permite confiar en la visión que está a la base de la toma de decisiones de las altas esferas de la jerarquía, ya sea empresarial o política. Esta herramienta es el alcance temporal de la discreción llamado también horizonte temporal (time-span of discretion), con la cual se mide qué tan lejos puede un ser humano visualizar correctamente y lograr completar un trabajo en el futuro. Según Jaques, el horizonte temporal de un director ejecutivo de estrato siete abarca un período mínimo de entre $20 \mathrm{y}$ 50 años, y corresponde a los CEO (Chief Executive Officer) de grandes empresas multinacionales y, por supuesto, a los jefes de Estado.
El propósito de este estudio fue identificar en el análisis del discurso de los CEO de los estratos seis a ocho (en tanto que son los responsables de la dirección ejecutiva), los elementos comunes y pautas del pensamiento acerca de las realidades económicas y sociales del futuro en donde sus empresas están invirtiendo, y contrastarlo con el panorama visualizado por especialistas en analizar las tendencias globales, tales como Jeremy Rifkin y sus postulados de la tercera revolución industrial (Rifkin, 2013); Alex Pentland y la física social (Pentland, 2014); Andrés Oppenheimer (2018) y el futuro del trabajo en la era de la automatización; y Ray Kurzweil (1999), fundador de la Universidad de la Singularidad, en su obra La era de las máquinas espirituales.

Aunado a lo anterior, fue necesario identificar en el discurso de los CEO los elementos comunes y temas de análisis recurrentes, asícomo los tópicos de importancia para el futuro económico de la sociedad, y así compararlos con la visión de los expertos.

\section{REVISIÓN DE LA LITERATURA}

\section{El papel del CEO en la organización}

El CEO, en una organización lucrativa, es la máxima autoridad jerárquica con carácter ejecutivo que dispone de la autoridad formal para dar órdenes y tomar decisiones operativas, y al mismo tiempo es responsable de rendir cuentas al Consejo Directivo por su gestión y por el trabajo de sus subordinados (Jaques, 2006). No se debe confundir con el presidente del Consejo (Chairman of the Board), quien preside una asociación llamada Consejo Directivo, en donde todos los miembros tienen voz y voto, y no existe jerarquía entre ellos. 
El Consejo Directivo es el encargado de designar al CEO y supervisar y valorar el ejercicio de sus funciones. Las funciones de ambos directivos son de una naturaleza diferente, la del presidente del Consejo es de autorizar y pedir cuentas, la del CEO es de operar una organización jerárquica, presentar proyectos, solicitar autorizaciones y rendir cuentas. Sin embargo, comenta Kakabadse (2006) que en varios países como los Estados Unidos de Norteamérica, Francia, Bélgica y parte de Canadá, es común que una misma persona ocupe simultáneamente estos dos puestos que son de naturaleza diferente.

\section{La personalidad del CEO}

El análisis del discurso corporativo expresado por el CEO está ganando la atención de los investigadores (Dewan, 2017). Según el autor, este método facilita la profundidad de análisis y tiene aplicación en las narrativas corporativas. Por ejemplo, Patelli y Pedrini (2014) exploran la sinceridad del tono retórico de 664 comunicaciones de los CEO al Consejo Directivo, y su asociación con el desempeño de la empresa y afirman que el tono optimista es congruente con el desempeño pasado y futuro. Sin embargo, en situaciones extremas como es el caso de un accidente en una planta nuclear, Beelitz y Merkl-Davies (2012) concluyen que la narrativa corporativa es un medio para consolidar el interés privado de las corporaciones, más que de incrementar la transparencia y la rendición de cuentas.

Hyland (1998) analizó 137 cartas de CEO en donde se revela la naturaleza esencialmente retórica del estilo de comunicación del CEO. El autor encontró evidencias de un metadiscurso en la comunicación en los negocios y apunta hacia la necesidad de una retórica consciente de este género persuasivo. La narrativa de los CEO está compuesta de varios textos simbólicos que guían o dirigen hacia la forma en que deben entender, evaluar y responder a la información.
En cuanto a los valores del CEO, Cardo \& Guerci (2018) establecen tres tipos de orientación de los directivos que abrazan la sustentabilidad en sus empresas. En primer lugar, están aquellos que se orientan a los negocios por oposición a quienes se orientan hacia los valores; luego, los directivos orientados al interior de la organización vs. los que se orientan hacia el exterior; y, finalmente, los que tienen visión a corto plazo vs. los de visión a largo plazo.

Al revisar la historia de una gran organización, Paroutis, McKeown \& Collinson (2013) encontraron que los CEO con frecuencia recurren al mito, que es una analogía usada para explicar lo inexplicable, y darle un sentido al caos. Encontraron en el nivel analítico cómo el CEO buscaba legitimar su discurso y cómo los actores respondieron al discurso. A lo largo de 27 años hubo en la empresa varios tipos de discurso. Primero se dio el discurso de la calidad, en la época en que los japoneses irrumpieron en el mercado con productos de calidad; después llegó el discurso de la reingeniería, cuando se percataron de que las organizaciones se estaban volviendo obsoletas para reducir costos; y, finalmente, vino la época del discurso de la reinvención.

Un discurso que ha sido ampliamente empleado es el de la estrategia. $\mathrm{Al}$ analizar diversas investigaciones enfocadas en los CEO exitosos, Morton, Stacy \& Mohn (2018) han encontrado una nueva variante del enfoque estratégico, la agilidad estratégica, la cual definen como "la práctica de ajustar continuamente y adaptar la dirección estratégica del núcleo del negocio en un flujo de praxis estratégica a través del tiempo, como una función de las ambiciones estratégicas y de las circunstancias cambiantes" (p. 96).

A muchos llama la atención el lenguaje triunfante y hasta cierto punto arrogante con que los CEO comunican proyectos o sus resultados; Craig \& Amernic (2018) analizaron 23 cartas firmadas por CEO supuestamente 
arrogantes. El programa empleado por los investigadores registró su discurso como "realismo", lo cual no es distintivo de arrogancia. Por lo tanto, la generalidad de los CEO de larga carrera en las organizaciones no llega a la arrogancia. Un CEO arrogante tiene la inquebrantable creencia de que será reivindicado por Dios o por la historia, es incansable, temerario e impulsivo, y tiende a poner por encima de la práctica, de los costos y de los resultados su visión amplia de la rectitud moral.

Si bien la generalidad de los CEO no cae en la arrogancia, según Reilly, Roer, Caldwell \& Chatman (2013), el discurso del CEO con frecuencia está permeado por la grandiosidad del poder que acumula y por el narcisismo, que es dominio, autoconfianza, grandiosidad y baja empatía. Los CEO narcisistas pueden tomar decisiones impulsivas riesgosas, tener paquetes de compensación mayores que los de sus homólogos menos narcisistas, y esas diferencias se incrementan entre mayor es el tiempo que el ejecutivo permanece al frente de la organización. Las organizaciones manejadas por CEO narcisistas experimentan considerables caídas, incluyendo evidencia de incremento en la toma de decisiones riesgosas, pagos exagerados por adquisiciones, manipulación de datos contables, que pueden llegar al fraude (Reilly, Doer \& Chatman, 2017). Los CEO también pueden padecer de neofilia, que es un amor fetichista por todo lo novedoso (Hodge, 2010), se emocionan con la novedad, para ellos lo nuevo es virtud y una meta por alcanzar.

Hasta ahora ha quedado claro que el CEO ejerce el liderazgo en la organización; la mayoría de los artículos de investigación en el tema de liderazgo tienen como fuente de información al CEO. Es así que Kariskova, Green \& Le Breton (2013), al analizar el lado obscuro del liderazgo de los CEO, hicieron una revisión de lo que dieron por llamar el liderazgo destruction, que en su definición habla de acciones emanadas del comportamiento de un líder que intenta dañar a la organización o a sus seguidores, y que se manifiesta de múltiples formas, tales como encaminar a los seguidores a que establezcan metas en contra de los intereses legítimos de la empresa, o emplear un estilo de liderazgo con métodos dañinos de influencia sin importar las justificaciones de tal comportamiento.

Con todo lo anterior, es de temer que la organización experimente la llegada de un CEO con personalidad inapropiada para tratar la gravedad de los problemas que a diario se enfrentan. No obstante, los CEO que perduran y dan buenos resultados son aquellos que, entre otras virtudes, tienen una habilidad cognitiva general (GCA) (Reilly \& Chatman, 1994). Esta habilidad sirve para predecir un buen desempeño tanto en el trabajo como en las situaciones y las carreras.

Más aún, otros investigadores como Ou, Tsui, Kinicki, Waldman, Xiao \& Jiwen Song (2014) han descubierto el lado de la sabiduría del CEO, en lo que han dado por llamar el lider humilde, y que se refiere a la integridad y a los valores morales de la persona, así como su expresión ética ante las complejidades de su tarea.

\section{El alcance temporal de la discreción}

Una característica esencial para la idoneidad en el puesto del CEO es el alcance temporal de la discreción, un tipo de inteligencia que no es el IQ, y que está involucrada en el tiempo máximo que una persona visualiza para completar su tarea más larga sin dirección y utilizando su criterio (Jaques, 2006). Entre más largo es el alcance temporal, más elevado es el nivel de trabajo. Entre más largo es el tiempo en que una persona dispone de autonomía sin que sus resultados sean evaluados, mayor es el esfuerzo psicológico requerido por la tarea. A mayor tiempo de soportar la incertidumbre y aun asícontinuar con el trabajo, mayor es la responsabilidad. A mayor tiempo que una organización deja ejercer la autonomía de un individuo, tomar decisiones y destinar recursos, más grande es la confianza que se le tiene y mayor es la responsabilidad que se le confiere (Jaques et al., 2013). 
Los estudios de Jaques y Cason a través de los años demostraron que el alcance temporal tiene una estrecha correlación $(\mathrm{r}=0.95)$ con la capacidad de procesamiento mental. Esta última se manifiesta en el manejo de la complejidad del orden de información, que puede ser concreto, simbólico, abstracto-conceptual o universal. A su vez, cada orden dispone de cuatro métodos de procesamiento mental, que son declarativo, acumulativo, serial y paralelo. En la tabla 1 se muestra la pirámide del procesamiento mental, en cuya base aparece el orden concreto con el método declarativo, caracterísitco de los niños pequeños, y en la cima está el orden universal, con el método paralelo, característico de unos pocos privilegiados a quienes se denomina genios (Jaques et al., 2013).

Tabla 1. Categorías de complejidad del proceso mental

\begin{tabular}{|c|c|c|}
\hline Categoría & $\begin{array}{l}\text { Complejidad } \\
\text { del orden de la } \\
\text { información }\end{array}$ & $\begin{array}{c}\text { Método de proceso } \\
\text { mental }\end{array}$ \\
\hline D4 & $\begin{array}{c}\text { Cuarto orden } \\
\text { universal }\end{array}$ & Paralelo \\
\hline D3 & " & Serial \\
\hline D2 & $"$ & Acumulativo \\
\hline D1 & $"$ & Declarativo \\
\hline C4 & $\begin{array}{c}\text { Tercer orden } \\
\text { abstracto-conceptual }\end{array}$ & Paralelo \\
\hline C3 3 & $"$ & Serial \\
\hline $\mathrm{C} 2$ & " & Acumulativo \\
\hline C1 & " & Declarativo \\
\hline B4 & $\begin{array}{l}\text { Segundo orden } \\
\text { simbólico }\end{array}$ & Paralelo \\
\hline B3 & " & Serial \\
\hline B2 & “ & Acumulativo \\
\hline B1 & " & Declarativo \\
\hline A4 & $\begin{array}{l}\text { Primer orden } \\
\text { concreto }\end{array}$ & Paralelo \\
\hline $\mathrm{A} 3$ & " & Serial \\
\hline $\mathrm{A} 2$ & “ & Acumulativo \\
\hline $\mathrm{A} 1$ & $"$ & Declarativo \\
\hline
\end{tabular}

Fuente: Jaques \& Cason (2013, p. 35).
$\mathrm{Al}$ reunir la capacidad de procesamiento mental con el alcance temporal de la discreción, se obtiene el cuadro de la teoría de los sistemas estratificados, mediante el cual se coloca a la persona correcta en el puesto correcto dependiendo de su nivel de trabajo, tal como se muestra en la tabla 2. Adicionalmente, los estudios de este autor indican el nivel salarial equitativo para cada estrato.

Tabla 2. Patrones de estratos de la organización requerida

\begin{tabular}{|c|c|c|c|c|}
\hline Estrato & $\begin{array}{l}\text { Alcance } \\
\text { temporal }\end{array}$ & $\begin{array}{c}\text { Capacidad de } \\
\text { procesamiento } \\
\text { mental }\end{array}$ & Nivel jerárquico & $\begin{array}{c}\text { Paga } \\
\text { Equitativa }\end{array}$ \\
\hline VIII & 50 años & $\begin{array}{l}\text { C4 Abstracto } \\
\text { conceptual } \\
\text { paralela }\end{array}$ & $\begin{array}{l}\text { Ceo } \\
\text { multinacional }\end{array}$ & $32 x$ \\
\hline VII & 20 años & $\begin{array}{l}\text { C3 Abstracto } \\
\text { conceptual } \\
\text { serial } \\
\end{array}$ & $\begin{array}{l}\text { Ceo } \\
\text { corporativo }\end{array}$ & $16 \mathrm{x}$ \\
\hline VI & 10 años & $\begin{array}{l}\text { C2 Abstracto } \\
\text { conceptual } \\
\text { acumulativa }\end{array}$ & $\begin{array}{l}\text { Vicepresidente } \\
\text { ejecutivo }\end{array}$ & $8 \mathrm{x}$ \\
\hline V & 5 años & $\begin{array}{l}\text { C1 Abstracto } \\
\text { conceptual } \\
\text { declarativa }\end{array}$ & $\begin{array}{l}\text { Director de } \\
\text { unidad de } \\
\text { negocios }\end{array}$ & $4 x$ \\
\hline IV & 2 años & $\begin{array}{l}\text { B4 Simbólica } \\
\text { paralela }\end{array}$ & $\begin{array}{l}\text { Gerente de } \\
\text { planta }\end{array}$ & $2 \mathrm{x}$ \\
\hline III & 1 año & $\begin{array}{l}\text { B3 Simbólica } \\
\text { serial }\end{array}$ & $\begin{array}{l}\text { Gerente de } \\
\text { Área }\end{array}$ & X \\
\hline II & 3 meses & $\begin{array}{l}\text { B2 Simbólica } \\
\text { acumulativa }\end{array}$ & $\begin{array}{l}\text { Gerente de } \\
\text { Línea }\end{array}$ & $55 x$ \\
\hline I & 1 día & $\begin{array}{l}\text { B1 Simbólica } \\
\text { declarativa }\end{array}$ & Operario & $.31 \mathrm{x}$ \\
\hline
\end{tabular}

Fuente: Jaques (2006, p. 41).

\section{La tercera revolución industrial}

Algunos autores en la actualidad han tomado el papel de sondear el futuro, tal como lo hizo Alvin Toffler (1979) en los años setenta, cuando presentó serias reflexiones acerca de las nuevas realidades de la sociedad. 
En la actualidad, uno de los estudiosos prácticos de las tendencias económicas y que ha tenido éxito al ser escuchado en la comunidad europea es Jeremy Rifkin, economista y promotor del capitalismo colaborativo.

Según Rifkin, el capitalismo seguirá formando parte del panorama social, pero el autor pone en duda su prevalencia como paradigma económico dominante durante la segunda mitad del siglo XXI (Rifkin, 2016). Estamos presenciando los primeros trazos de la economía colaborativa, gracias a una paradoja del capitalismo, que en su afán de productividad y reducción de costos ha llegado al costo marginal cero, lo que hace que "millones de jóvenes de todo el mundo sean prosumidores (productores y consumidores a la vez) que comparten su propia música, vídeos, blogs, libros y otros servicios a un coste cercano a cero" (Rifkin, 2016) ${ }^{1}$.

Para el autor, la humanidad se encuentra a las puertas de la tercera revolución industrial (que no debe ser confundida con la cuarta revolución industrial del Foro Económico Mundial, en donde se examina la sociedad del conocimiento). Según Rifkin, una revolución industrial se da cuando convergen tres factores básicos de producción, que son la energía, la tecnología de comunicación y la tecnología de transporte.

En la tercera revolución Industrial, se hará el cambio a energías renovables, la tecnología de comunicación serán las redes digitales y la tecnología de transporte será el transporte eléctrico.

\section{La física social y el big data}

Alex Pentland es director del laboratorio de medios del MIT y dirige las iniciativas de big data y protección de datos personales en el Foro Económico Mundial. Para Pentland, el big data es una herramienta básica para entender a profundidad la realidad económica y social en una era de masas en donde la información se propaga de una manera vertiginosa. En su libro Física social (Pentland, 2014), el autor señala que muchas ideas que tenemos sobre nosotros mismos y la sociedad están equivocadas, y que no basta con tener ideas brillantes, pues aquellos que saben cosechar las ideas brillantes de otros son los que inciden en los cambios de la sociedad. Así pues, la física social es una ciencia social cuantitativa que pretende discernir la forma en que las interacciones sociales afectan las metas y las decisiones de los individuos. "Describe de forma confiable las conexiones matemáticas entre la información y el flujo de ideas, por un lado, y el comportamiento humano, por el otro; en la física social se conceptualiza a la sociedad como una inmensa máquina" (Pentland, 2014, p. 13).

Es de esperar pues que los CEO de grandes corporaciones hagan uso del big data que se produce en la operación diaria al interior de sus organizaciones y fuera de ellas, para sustentar la administración estratégica y toma de decisiones.

\section{El futuro del trabajo en la era de la automatización}

Son varios los autores que han incursionado en el tema de la automatización de las fábricas y el consiguiente desempleo de los obreros, el mismo Rifkin lo hizo en su momento. Por su parte, Andrés Oppenheimer abundó sobre el tema en su libro Sálvese quien pueda (Oppenheimer, 2018). Para este autor, llegará el fin, no solamente de muchos empleos operativos que requieren habilidades físicas repetitivas, que pueden ser desempeñados de manera más eficiente por la robótica; sino también están amenazados los empleados administrativos que pueden ser substituidos por la

1 El texto de la entrevista no tiene paginación. 
inteligencia artificial, o los vendedores en las tiendas que se perfilan a ser reemplazados por el comercio electrónico, entre otros.

Sin embargo, el autor señala que los empleos no se van a acabar con la aparición de la robótica y la inteligencia artificial, ya que habrá nuevas modalidades de empleo y oportunidades en áreas como la cibernética.

Habrá grandes cambios en el mundo laboral, principalmente en los empleos de manufacura, periodistas, restaurantes, supermercados y tiendas departamentales, empleados bancarios, abogados, contadores y aseguradores, médicos, docentes, transportistas, actores y deportistas.

Los empleos tendrán que ser reinventados.

La gente con altos niveles de habilidades o estudios estará bien equipada para moverse hacia los nuevos trabajos que surjan en los próximos años, mientras que los que están menos capacitados serán los que corren más riesgo de ser reemplazados por completo. (Oppenheimer, 2018, p. 21)

El autor señala diez áreas que tendrán gran potencial de trabajo en el futuro, a saber: los asistentes de salud, los analistas de datos, ingenieros de datos y programadores, los policías digitales, los asesores de ventas, los cuidadores y programadores de robots, los profesores y maestros con nuevos perfiles, los especialistas en energías alternativas, los artistas, deportistas y creadores de entretenimiento, los creadores y diseñadores de contenidos comerciales, y los consejeros espirituales.

\section{MÉTODO}

Para la realización del estudio se empleó un método cualitativo con la recolección de textos cuyo contenido fuera susceptible de análisis, con la finalidad de dar respuesta al objetivo de contrastar la visión de los expertos en temas del futuro, con la de quienes toman decisiones tendientes a la creación del futuro económico de la sociedad.

\section{Recolección de datos}

La recolección de datos se llevó a cabo con la búsqueda de declaraciones de los ejecutivos tomadas de entrevistas a diferentes medios de comunicación del ámbito de los negocios. La unidad de análisis fue la persona del CEO, y posteriormente su discurso fue dividido en unidades de pensamiento. En este sentido, la desventaja de información "permeada" se anula, ya que es el entrevistado el objeto de estudio (Hernández-Sampieri, Fernández \& Bautista, 2010).

Todas las fuentes utilizadas son ampliamente conocidas en el ámbito empresarial y cuentan con larga trayectoria de entrevistas a capitanes de empresas. Se tomó en cuenta el listado de las más grandes multinacionales de la revista Fortune, ya que estas organizaciones tienen gran influencia en la población a nivel global y realizan una parte substancial de la investigación científica orientada a la producción de tecnología que impacta al resto de las empresas en el mundo. Quince de los 24 CEO que conformaron la muestra dirigen alguna de las primeras 40 organizaciones del listado. También se consideraron los directivos de las más grandes empresas mexicanas. Otro criterio de selección fue la búsqueda en ese mismo listado de fundadores y dueños que hayan ocupado el puesto de CEO en algún período de su trayectoria laboral. Un cuarto criterio de selección fue el de directivos cuyo distintivo es la innovación con productos y servicios disruptivos de alcance mundial.

Se identificaron y se recabaron entrevistas y declaraciones de $\mathrm{CEO}$ que cumplieron con los requisitos 
anteriores. Los textos de las entrevistas fueron revisados con la eliminación de la intervención del entrevistador, dejando solamente la parte de la respuesta del entrevistado para convertirlos en un discurso fluido. Tratándose de discursos, se tomó el texto integral sin revisión alguna.

\section{Procesamiento de datos}

El análisis del discurso completo que en total cubrió 110 cuartillas que contienen exclusivamente las respuestas de los CEO se realizó por unidades de pensamiento según el método descrito por Herzberg (1959). La razón de usar este método responde a la inquietud de buscar el metadiscurso (Hyland, 1998), el cual va más allá de la materia en cuestión y señala la presencia del autor, en donde se revela a sí mismo y sus propósitos comunicativos. Se requiere analizar una unidad después de otra para obtener categorías de metadiscurso con intenciones de influencia en el futuro económico de la sociedad.

En total, se analizaron 430 unidades de pensamiento obteniendo 172 categorías correspondientes a 18 temas principales que están englobados en los seis temas centrales que serán objeto de análisis en la sección de resultados, a saber: la persona del CEO, lecciones de gestión empresarial, gobiernos vs. empresas, la innovación tecnológica, el alcance temporal de la sociedad en su conjunto, y el medio ambiente.

Para responder a la inquietud del investigador acerca de si existe paralelismo entre la orientación del CEO y la visión de los gurús que predicen el futuro económico, se siguió el método de la teoría fundamentada, de comparaciones constantes (Glaser \& Strauss, 1967/2006).
Tabla 3. Visiones de futuro económico de la sociedad

\begin{tabular}{|c|c|}
\hline Autor & Visión \\
\hline Jeremy Rifkin (2016) & $\begin{array}{l}\text { - Capitalismo colaborativo } \\
\text { - Energías renovables generadas in situ } \\
\text { - Internet de las cosas } \\
\text { - Parque vehicular eléctrico }\end{array}$ \\
\hline Alex Pentland (2014) & $\begin{array}{l}\text { - Uso de big data para entender a la } \\
\text { sociedad y predecir eventos masivos y } \\
\text { cambios en normas sociales }\end{array}$ \\
\hline $\begin{array}{l}\text { Andrés Oppenheimer } \\
\text { (2018) }\end{array}$ & $\begin{array}{l}\text { - Uso generalizado de la robótica en la } \\
\text { vida económica y social } \\
\text { - Nuevas formas de trabajo para los } \\
\text { seres humanos }\end{array}$ \\
\hline $\begin{array}{l}\text { Ray Kurzweil (Singularity } \\
\text { University) (1999) }\end{array}$ & $\begin{array}{l}\text { - Vehículos autónomos. La mayor } \\
\text { parte de la conducción estará en manos } \\
\text { de sistemas inteligentes no humanos } \\
\text { para } 2030 \\
\text { - Robots domésticos utilizados } \\
\text { masivamente para } 2030 \\
\text { - Mayor parte de la actividad } \\
\text { intelectual del planeta será realizada } \\
\text { por inteligencia artificial para } 2050 \\
\text { - Implantes cerebrales permitirán } \\
\text { conenctar nuesto cerebro directamente } \\
\text { a la red: mundo virtual, funciones } \\
\text { extra, aumento de memoria o } \\
\text { inteligencia antes de } 2050 \\
\text { - Guerras hambres y pobreza } \\
\text { desaparecerán gracias al desarrollo } \\
\text { tecnológico antes de } 2050 \\
\text { - Inteligencia artificial conciente } \\
\text { - Nanotecnología transformará al } \\
\text { mundo }\end{array}$ \\
\hline
\end{tabular}

Fuente: elaboración propia.

En cuanto al perfil de los CEO en lo que respecta al alcance temporal de la discreción, se realizó el análisis del discurso mediante el método descrito por Jaques et al. (2013), cuyo resultado aparece en la tabla 4. Este método hace hincapié en que el discurso revela el método de procesamiento de la información. 
Si el discurso es por frases aisladas, sueltas y sin relación unas con otras, entonces el método es declarativo. Si al hablar el entrevistado hace mención a un argumento y lo sustenta con la suma de muchas y variadas proposiciones, entonces el método es acumulativo. Si, por el contrario, se acude a la hilación de causa y efecto, el método es serial, y cuando dos o más series se entrelazan unas con otras (si y solo si) el método es paralelo. En cuanto al orden de complejidad de la información, todos los CEO han dejado atrás el orden simbólico de los términos y utilizan el orden abstracto conceptual, que es el orden de las ideas y las teorías complejas, y unos pocos han llegado al orden de los universales.

Tabla 4. Estrato, capacidad de procesamiento mental y alcance temporal de la discreción de la muestra de estudio

\begin{tabular}{|c|l|c|c|}
\hline Estrato & $\begin{array}{c}\text { Capacidad de } \\
\text { procesamiento } \\
\text { mental }\end{array}$ & $\begin{array}{c}\text { Alcance } \\
\text { temporal años }\end{array}$ & $\begin{array}{c}\text { Número de } \\
\text { CEO }\end{array}$ \\
\hline X & $\begin{array}{l}\text { D2 Universal } \\
\text { acumulativo }\end{array}$ & $200+$ & 1 \\
\hline IX & $\begin{array}{l}\text { D1 Universal } \\
\text { declarativo }\end{array}$ & $100+$ & 4 \\
\hline VIII & $\begin{array}{l}\text { C4 Abstracto } \\
\text { conceptual paralelo }\end{array}$ & $50-100$ & 7 \\
\hline VII & $\begin{array}{l}\text { C3 Abstracto } \\
\text { conceptual serial }\end{array}$ & $20-50$ & 4 \\
\hline VI & $\begin{array}{l}\text { C2 Abstracto } \\
\text { conceptual } \\
\text { acumulativo }\end{array}$ & $10-20$ & 8 \\
\hline
\end{tabular}

Fuente: elaboración propia.

\section{RESULTADOS}

La lectura de las unidades de pensamiento en busca del metadiscurso reveló puntos importantes en la postura y dimensión del pensamiento de los CEO. Todos los discursos analizados dan fe de una visión global, no solamente en cuestión de fronteras nacionales, sino de retos y problemas que enfrenta la humanidad en el ámbito político, social y ambiental. El CEO se coloca por encima de su localidad de origen, así sea Bentonville 0 Nueva York, Silicon Valley, o la Ciudad de México. Es un ciudadano del mundo, poseedor de una cultura universal que trasciende fronteras y creencias, precursor de una sociedad unificada, pero sin la pérdida de la propia identidad. El CEO se mueve a su antojo alrededor del planeta y no tiene preferencias ni aborrecimientos por tal o tal lugar, simplemente observa la pluralidad de razas y culturas. Esta altura de visión le permite encontrar las interrelaciones de la actividad incesante de la humanidad, en donde su empresa juega un papel destacado en la producción de riqueza.

El análisis del discurso extrajo seis temas principales que están en la mente de los CEO y que aparentemente cimientan su visión de futuro y su actuación a corto plazo en el ámbito de la organización. Estos temas tienen que ver, en primer lugar, con la naturaleza de la institución CEO, con la personalidad de los ocupantes del puesto, su perfil, sus funciones, el oficio de ser $\mathrm{CEO}$ y su evaluación. También se refieren, en segundo lugar, a las lecciones de management que nos ofrecen, particularmente en el ámbito de la estrategia (una prerrogativa del CEO, que orquesta la actuación de toda la organización), así como de la cultura organizacional y su importancia en la expansión de la empresa más allá de sus fronteras.

El tercer tema central tiene que ver con la innovación tecnológica como pieza indispensable en la creación y conducción de las empresas, con sus efectos al interior de la organización y sus efectos actuales y futuros en la sociedad. Asimismo, se abordan en cuarto lugar los problemas graves en política, educación y salud, temas que no han sido resueltos en la sociedad y que pueden ser atribuidos más que a los gobiernos, a un bajo promedio de alcance temporal de la sociedad en su conjunto. 
Finalmente, cabe señalar dos temas relacionados que tienen que ver con la naturaleza del gobierno y la naturaleza de la empresa, y con el papel de ambos en el asunto del cambio climático.

El metadiscurso revela claramente que para todos los CEO estudiados no existe duda en que actúan dentro de la realidad de la economía de mercado y de la libre empresa, como resultado del capitalismo que se desarrolló en el siglo XVIII en Inglaterra y que ha ido evolucionando hasta convertirse en el capitalismo del mundo tecnológico hiperconectado de los grandes conglomerados, de las fusiones y adquisiciones, y sin perder su esencia de propiedad privada y acumulación de capital, con la obtención de utilidades como meta primordial de la organización. Es en esta realidad que el pensamiento de los CEO aborda el problema de la relación empresa-gobierno, como dos colosos que se disputan el poder y que se han visto obligados a negociar su naturaleza y facultades en aras de una sana convivencia.

Tabla 5. Esquema de análisis del discurso del CEO

\begin{tabular}{|c|c|c|c|}
\hline $\begin{array}{l}\text { Unidad de } \\
\text { pensamiento }\end{array}$ & Categoría & Temas & $\begin{array}{c}\text { Tema } \\
\text { central }\end{array}$ \\
\hline 116 & 45 & $\begin{array}{l}\text { - Naturaleza y } \\
\text { funciones del puesto } \\
\text { - Perfil del puesto } \\
\text { - Oficio de ser CEO } \\
\text { - Evaluación de ser } \\
\text { CEO }\end{array}$ & $\begin{array}{l}\text { La persona } \\
\text { CEO }\end{array}$ \\
\hline 98 & 41 & $\begin{array}{l}\text { - Pensamiento } \\
\text { estratégico } \\
\text { - Cultura } \\
\text { organizacional }\end{array}$ & $\begin{array}{l}\text { Lecciones } \\
\text { de gestión } \\
\text { empresarial }\end{array}$ \\
\hline 89 & 39 & $\begin{array}{l}\text { - Naturaleza de los } \\
\text { gobiernos } \\
\text { - Funciones de los } \\
\text { gobiernos } \\
\text { - Naturaleza de las } \\
\text { empresas } \\
\text { - Funciones de las } \\
\text { empresas }\end{array}$ & $\begin{array}{l}\text { Gobierno } \\
\text { os. empresas }\end{array}$ \\
\hline
\end{tabular}

\begin{tabular}{|c|c|l|l|}
\hline $\begin{array}{c}\text { Unidad de } \\
\text { pensamiento }\end{array}$ & Categoría & \multicolumn{1}{|c|}{ Temas } & $\begin{array}{c}\text { Tema } \\
\text { central }\end{array}$ \\
\hline 69 & 28 & $\begin{array}{l}\cdot \text { Fundamentos de la } \\
\text { innovación tecnológica } \\
\cdot \text { Efectos en las } \\
\text { empresas } \\
\cdot \text { Efectos en la } \\
\text { sociedad }\end{array}$ & $\begin{array}{l}\text { Innovación } \\
\text { tecnológica }\end{array}$ \\
\hline 40 & 11 & $\begin{array}{l}\text {-Solución a problemas } \\
\text { graves de la sociedad } \\
\cdot \text { Minoría con elevado } \\
\text { alcance temporal }\end{array}$ & $\begin{array}{l}\text { Alcance } \\
\text { temporal de } \\
\text { la sociedad }\end{array}$ \\
\hline 18 & 8 & $\begin{array}{l}\cdot \text { La defensa del medio } \\
\text { ambiente } \\
\cdot \text { Propuesta de } \\
\text { soluciones } \\
\cdot \text { Primacía de las } \\
\text { utilidades } \\
\cdot \text { Complejidad }\end{array}$ & $\begin{array}{l}\text { Medio } \\
\text { ambiente }\end{array}$ \\
\hline
\end{tabular}

Fuente: elaboración propia.

\section{Lecciones de gestión empresarial}

A lo largo de la narrativa de estudio se encontraron gran cantidad de acciones propias de la gestión empresarial y del liderazgo, lo cual concuerda con la aseveración de Mintzberg de que la administración no es una ciencia, sino una práctica (Mintzberg, 2018). Al observar la práctica ejercida por los $\mathrm{CEO}$ nos damos cuenta de que su actividad al frente de la empresa está saturada de lecciones que pueden inspirar a los profesionales de la administración. Casi todo lo expresado en las entrevistas y declaraciones habla de estrategia y de cultura. La estrategia aparece con mayor frecuencia y la describen como el mapa de ruta para lograr la visión. Ahora bien, para crear la estrategia es necesario encontrar la identidad central y el propósito de la empresa, lo cual permite actualizar la visión en la naturaleza del negocio, concepto que se repite una y otra vez en gran parte del texto. De esta manera, los $\mathrm{CEO}$ de empresas petroleras tienen muy claro que ellos se encuentran en el sector de la energía, 
y tienen listos los escenarios de la introducción de otras energías para participar en esos mercados.

De igual manera, para Akio Toyoda, su empresa no es fabricante de autos, es una empresa dedicada a la movilidad. También lo expresan como "enfocarse en el núcleo del negocio y encontrar la disrupción".

Así pues, al contar con un propósito claro y metas derivadas de la estrategia, se deben tomar decisiones con pensamiento sistémico que permitan conseguir adquisiciones estratégicas y asociaciones con aliados de primer nivel aportando tecnología de punta. Lo anterior queda muy claro en el caso de empresas del sector de comunicación. AT\&T ha hecho las adquisiciones necesarias para tener contenidos y participar plenamente en los mercados de la comunicación y el entretenimiento. Estas decisiones pueden ser de carácter incremental o bien de carácter revolucionario (disruptivas), siendo estas últimas las más favorecidas por los entrevistados. La gran mayoría enfatiza la importancia del gobierno corporativo para el buen desempeño, para contar con una oferta de servicios a la medida y no perder el control de los suministros, aun cuando Elon Musk manifiesta ser accionista mayoritario y tener pleno control de sus organizaciones.

El segundo tema que resalta en la práctica de la gestión empresarial es la filosofía que debe estar a la base de la cultura organizacional. Esta filosofía habla de empoderamiento de la capacidad de los colaboradores, de la organización como un lugar de aprendizaje y realización en la carrera de los empleados, de lograr una cultura en donde la gente le tenga amor a su trabajo y cariño por la compañía. Las declaraciones de Lorenzo Servitje, Satya Nadela, Elon Musk, Larry Page, Dough Mc Millon (quien manifiesta estar capacitando a sus empleados para la transición hacia los empleos del futuro) y Darren Woods en este sentido son impactantes, ya que no dejan duda de que no responden a pose alguna, sino a la realidad que se esfuerzan por plasmar en sus organizaciones. Aun cuando no está expresado de manera explícita por los directivos arriba citados, el lector puede percatarse de que en un mundo globalizado estas empresas multinacionales terminarán por fusionar culturas hacia el nacimiento de una cultura multinacional unificada, con ciudadanos del mundo. En empresas como Motorola, su $\mathrm{CEO}$ asegura que esto ya es una realidad.

\section{Gobiernos y empresas}

Para los directivos de empresas que manejan grandes cantidades de recursos, y que por lo tanto figuran como entidades de poder en la sociedad, queda muy claro que existe otra entidad que maneja una mayor cantidad de recursos y tiene el poder de regular las actividades de las primeras. En todos los relatos se hace mención al papel del gobierno, ya sea como ente regulador o como ente redistribuidor de la riqueza. En ellos se manifiesta que la naturaleza del gobierno es gobernar con autoridad sobre todos los ciudadanos, dando por sentado que existe un contrato social implícito que le otorga esa facultad (Rousseau, 1989). Esta facultad, sin embargo, les quita privacidad a los ciudadanos en aras de la seguridad. A diferencia de la empresa, el gobierno no ha sido diseñado para ser eficiente, no es una de sus preocupaciones. Su principal función es la de garantizar la seguridad de los ciudadanos, seguida de la redistribución de la riqueza y el combate a la pobreza. Tiene también otras funciones que derivan de su naturaleza, como la responsabilidad por la salud y educación, responsabilidad para proveer servicios públicos. Regula las actividades de las empresas como parte de la correcta conducción de la economía. El gobierno canaliza fondos para la investigación básica y para todo tipo de actividades que rebasen la capacidad del sector privado.

Por su parte, la naturaleza de la empresa está en ser creadora de riqueza, ya que el gobierno no crea la riqueza, y por lo tanto está también en la naturaleza de 
la empresa la creatividad, la eficiencia, la creación del mundo del futuro. La empresa genera valor para sus accionistas y para todos los grupos de interés que se relacionan o son parte de ella, ya que es la poseedora del conocimiento profundo y del capital. Participa en las acciones del gobierno en favor de la sociedad a través de la filantropía, y, en este sentido, algunos de los entrevistados describen con detalle sus obras a favor de grupos marginados o sociedades económicamente muy atrasadas, ayudando a devolverles la salud y proporcionando educación. Emprender proyectos para solucionar problemas de la sociedad es una tradición dentro del mundo capitalista, así como la consigna no escrita de producir bienes y servicios de manera ética evitando negocios nocivos. Promover el desarrollo a largo plazo y responder a problemas sociales aparece como una constante en la mayoría de los relatos.

\section{Innovación tecnológica}

Los fundamentos de la innovación tecnológica, que es el cuarto tema en frecuencia de mención, se basan en la automatización de las actividades de producción, ya sea en el piso de fabricación o en la oficina de finanzas. No se menciona explícitamente a la labor de los robots que desempeñan las labores propias de los seres humanos ni se plantea esta problemática; parece ser una realidad con la cual se ha estado viviendo durante muchos años y gradualmente las empresas y los trabajadores se han ido adaptando a ella.

En el campo de las TI se mencionan con detalle los avances en conectividad, con la tecnología cibernética $5 \mathrm{G}$ con conexión inalámbrica, como un desarrollo tecnológico disruptivo que modificará rotundamente la latencia y capacidad de descarga que permitirán, entre otras cosas, el advenimiento de los vehículos autónomos en las calles de las ciudades. Se menciona también el desarrollo gradual de la tecnología de inteligencia artificial y de las máquinas que aprenden.
Los efectos de la innovación tecnológica en las empresas desatan posiciones encontradas entre los CEO estudiados. Por un lado, Akio Toyoda, CEO de Toyota, asegura que este período de cambio tecnológico que está viviendo la industria de la movilidad es un fenómeno que se da una vez en un siglo y que significará lograr la supervivencia, más que ser el ganador. Considera que es tiempo de realizar alianzas compartiendo tecnología (de hecho, Elon Musk manifiesta que Tesla tiene una alianza estratégica para compartir tecnología de autos eléctricos con Toyota y con Daimler). Por el contrario, Darren Woods, CEO de Exxon Mobil, asegura que gracias a las adquisiciones estratégicas en tecnología en la industria de la energía su empresa está lista para competir, no para sobrevivir, sino para ser la ganadora. Es probable que el contexto en que se mueven estas dos industrias sea tan diferente que permita que dos de sus participantes destacados en cada una de ellas sostengan posturas diametralmente opuestas.

Todos coinciden en que los cambios hasta ahora han sido graduales, aun cuando los directivos en sus escenarios ya tienen todo el panorama visualizado. De esta forma, pueden hacer uso de las tecnologías más avanzadas cuando se requieran, de acuerdo con la evolución de la sociedad y de los negocios.

Los efectos de la innovación tecnológica en la sociedad son mencionados por CEO de fondos de inversión o de servicios de información para inversionistas, como Larry Fink, Warren Buffett y Mike Bloomberg, quienes señalan la pérdida de empleos tradicionales como consecuencia del cambio tecnológico y también como un efecto negativo del libre comercio (por el cual están a favor), y sugieren que algo debe hacer la sociedad para resolver este problema.

Bill Gates manifiesta que en el reporte anual de la Fundación Gates aparece que para 2035 será un hecho la eliminación de la pobreza en el mundo gracias a la 
tecnología. Las declaraciones de Hans Vestberg, CEO de Verizon, sobre la necesidad de escalar la tecnología para lograr las metas de la $\mathrm{ONU}$ en cuanto a eliminación de la pobreza y mejoramiento de la salud, apuntan al mismo sentido.

Otros efectos de la tecnología en la sociedad son mencionados por Dough Mc Millon, CEO de Wal Mart, quien asegura que a través del planeta los mercados de venta al menudeo se están estandarizando, y que la tendencia es de cambio hacia el mercado digital sin que las tiendas físicas vayan a desaparecer. Hay muchas menciones del camino hacia el mundo virtual y de que la tecnología y la inteligencia artificial (IA) cambiarán para bien la vida de las personas, hasta el momento en que se déla unión de la IA con el ser humano.

\section{Alcance temporal de la sociedad}

Los dos últimos temas centrales tienen mucho en común, el alcance temporal de la sociedad y los problemas del medio ambiente están estrechamente relacionados. El ser humano se ha mostrado incapaz de mantener limpia su casa y, al contrario, parece que se ensaña en destruirla. A la sociedad en su conjunto le toma mucho tiempo realizar cambios, modificar sus modelos mentales para adaptarse a las nuevas realidades. Es esta seguridad experimentada en lo conocido la que ha impedido en el pasado la marcha hacia el futuro, y ahora con la destrucción de su hábitat el hombre ser verá obligado a emigrar del planeta, de ahíla visión de Elon Musk por colonizar Marte. Ya antes Karl Sagan hablaba de las épocas de obscurantismo que retrasaron por siglos la marcha de la humanidad (Sagan, 1980). El sentir de los CEO es que el sistema global está cambiando, pero toma mucho tiempo cambiarlo todo y en todas partes, pues a la sociedad en su conjunto le cuesta trabajo cambiar formas de pensar. Para Bill Gates, el problema del cambio climático y de otros problemas igual de graves no está tanto en si los gobiernos actúan y las empresas cooperan, sino en que el promedio del alcance temporal (horizonte temporal) de la gente en general es muy bajo.

Solamente una pequeña porción de la población puede dimensionar la magnitud de los problemas y es la que está levantando la voz, pero mientras el grueso de la población no lo haga los cambios no se podrán dar.

\section{Medio ambiente}

Ben van Beurden, CEO de Royal Dutch Shell, reseña el escenario de la empresa Shell respecto a alcanzar las metas del Acuerdo de París: triplicar la eficiencia energética, terminar con la deforestación, almacenamiento de baterías a gran escala, cambio a energías renovables, vehículos autónomos, vehículos basados en el mismo chasis estándar, todos los autos deben ser eléctricos cien por ciento en 2050.

Por su parte, Elon Musk enfatiza que hay que acelerar el advenimiento de las energías limpias para detener el juego loco de sacar carbono de la tierra para emitirlo a la atmósfera.

En general, los CEO afirman que la sustentabilidad es el deseo de los clientes, no importa los vientos políticos que soplen, la conciencia ambiental sigue en aumento. Las empresas desean estar a la vanguardia en investigación y tecnología para enfrentar el cambio climático. Los fabricantes de autos tienen la visión de cero choques, cero emisiones, cero congestiones.

El enfoque para solucionar los problemas del cambio climático debe ser de unión, coexistencia y colaboración, aunque coinciden en que los países ricos deben ser los catalizadores para que los demás países hagan algo respecto a la atmósfera.

Para avanzar en este tema es importante dejar de lado la lucha por las soluciones opuestas, todas las soluciones 
se deben adicionar para converger, aportar, y asíllegar a las metas propuestas, no una u otra, sino una más la otra. Se deben adicionar las mejoras al motor de combustión interna en cuanto a emisiones, en lo que se perfeccionan los autos eléctricos, así como usar biogás, y otros combustibles poco contaminantes. Bill Gates ha apostado por el perfeccionamiento de la energía nuclear con su empresa Terra Power, pues considera que las energías limpias están muy lejos en este momento de satisfacer por completo la demanda de energía.

El problema ambiental es sumamente complejo, porque, para empezar, la empresa capitalista tiene como principio la primacía de las utilidades sobre el compromiso con el medio ambiente, no obstante, las declaraciones de la mayoría de los CEO en el sentido de que las organizaciones lucrativas buscan protección del medio ambiente, y algunos señalan que su compromiso de responsabilidad social y sus acciones en favor de la sustentabilidad social y el entorno son poco conocidas.

Otras complejidades del problema apuntan en el sentido de que al sector energético se le está demandando más energía y al mismo tiempo menos emisiones y, por lo tanto, los objetivos sociales emergentes significan aumentar la producción de energía al mismo tiempo que se protege la naturaleza.

Gates señala que la paradoja de la riqueza se da cuando una sociedad pobre logra salir de esta condición. Entonces la gente pasa a formar parte de la clase media y demanda productos y servicios cuya producción impacta al medio ambiente.

El problema del cambio climático es la latencia, pasa tiempo antes de ver sus resultados, pero cuando se presentan crecen a una tasa exponencial.
El debate político acerca del cambio climático es un tema que distrae a la gente, pero las medidas que se han tomado no llegan ni por asomo a contrarrestar sus efectos negativos.

\section{CONCLUSIONES}

El propósito de este estudio fue de comprobar el grado de alineación de los CEO de empresas multinacionales con la visión de futuro de los especialistas en el tema, considerando que la naturaleza de la empresa es la innovación para crear el futuro.

En lo que se refiere a la visión de Rifkin, no se encontró evidencia de que los CEO tengan explícitamente la intención de migrar hacia un capitalismo colaborativo, al contrario, su visión apunta a proyectar a sus organizaciones como preponderantes en los mercados dentro de 50 años. Apple, Wal Mart y Exxon Mobil son las más enfáticas en este punto. Sin embargo, existe una voz discordante en la persona de Larry Fink, CEO del fondo de inversión Blackrock, quien en la carta a las empresas en donde su fondo invierte el dinero de sus inversionistas manifiesta que

el mundo está viviendo la mayor transferencia de riqueza en la historia: 24 billones de dólares que pasan de la generación de la posguerra, los baby boomers, a los millennials... Durante el año pasado vimos a algunos de los empleados más calificados del mundo abandonar sus labores en señal de protesta y participar en polémicas asambleas públicas para expresar su perspectiva en relación al propósito corporativo y su importancia. Este fenómeno continuará creciendo a medida que los millennials e incluso las generaciones más jóvenes ocupen cada vez más puestos de responsabilidad. En una encuesta reciente realizada por Deloitte, se les preguntó a los trabajadores millennials cuál debería 
ser el propósito principal del negocio, alcanzando un 63\% más de votos "mejorar la sociedad" que "generar rentabilidad". (Fink, 2019)

La mención de Fink acerca de un cambio de mentalidad en las nuevas generaciones, tanto de propietarios como de administradores de empresas, marca el preámbulo del debilitamiento del pensamiento emanado de la ética protestante en el sentido de considerar las ganancias como el motivador primordial y la meta única de la actividad empresarial, y por lo tanto se acerca a la postura de Rifkin y el capitalismo colaborativo.

En ningún relato se hace alusión a las impresoras 3D, ni al empoderamiento del ciudadano como resultado del costo marginal cero, que tampoco recibe menciones. No se visualiza al ciudadano común como generador de energía limpia in situ, en cada casa, en cada edificio, como postula Rifkin, ni de una red de energía que pueda ser compartida por todos. Los representantes del sector energía consideran a sus organizaciones como portadoras de la solución, no los consumidores. Cuando estén dadas las condiciones para dejar atrás el petróleo, sus empresas están ya listas para asumir el reto de producir y comercializar otro tipo de energías. De lo que síse habla en la mayoría de los relatos es de la hiperconectividad que menciona Rifkin, así como de la realidad que está a la puerta con el advenimiento del parque vehicular eléctrico, y en esto todos coinciden y están coordinando sus actividades para que suceda.

Para responder a otra de las preguntas de investigación relacionada con uno de los objetivos relativo a las inversiones de las empresas, se consultó la información de la revista Fortune de las 500 empresas más grandes del mundo (Fortune 500, 2019). Esta fuente revela que de las primeras 40 empresas de su listado, 8 son armadoras, 7 son petroleras, y 5 son fondos de inversión que en alguna medida fondean a las primeras. Lo anterior refuerza la aseveración de los CEO de que los cambios son graduales, y que el mundo del petróleo todavía tiene mucho que contar en la actividad económica.

En cuanto a la física social de Pentland, no se hace mención al big data, pero esta herramienta ya se usa para efectos de mercadotecnia y creación de escenarios. Bill Gates toca el punto en varias ocasiones, y dice que quedó atrás desde hace muchos años el tiempo en que el ciudadano no dejaba huella, que ahora la vigilancia de cámaras y los dispositivos electrónicos registran de manera continua la actividad de las personas. Para Gates, debe haber un debate en la sociedad acerca de las atribuciones que pueda tener el gobierno en este aspecto, ya que es muy fácil que los seres humanos pierdan su privacidad en aras de la seguridad nacional. Casi todos hacen alusión a las proyecciones de población para los próximos 50 años, y lo toman como un dato a tener en cuenta para adecuar sus proyectos ante esas realidades. Ninguno propone planes para intervenir el crecimiento poblacional ni para estabilizar el número de habitantes en las naciones. Tampoco se mencionan los fenómenos de migración causados por la política, las ideologías, o la pobreza, excepto para Gates, para quien es posible que algo malo ocurra en los próximos 50 o 100 años en el planeta, pero desea que no afecte de manera substancial la vida de las naciones.

Los empleos del futuro reseñados por Andrés Oppenheimer son ya una realidad en todas las empresas estudiadas, ellas mismas se han encargado de abrir esos puestos de trabajo y de capacitar a sus empleados para asumirlos. Parece ser que el problema está más a nivel del cambio que debe tener lugar en los hogares, en las escuelas y en las universidades; en la capacitación de los maestros que crecieron en otras realidades y en las acciones de gobierno que promuevan el uso de la tecnología por parte de sus ciudadanos de todos los estratos sociales.

Finalmente, las predicciones de Ray Kurzweil son también una realidad en las empresas. La robótica ya a nadie 
sorprende, los CEO casi no hacen alusión a ella, pues se da por hecho que así es ahora el trabajo en sus organizaciones, aunque ninguno menciona los robots domésticos de Kurzweil para el 2030. Los vehículos autónomos reciben la atención de los CEO de las empresas armadoras y de las de comunicación, y estos últimos explican con detalle la forma en que la tecnología $5 \mathrm{G}$ será indispensable para que esos autos puedan circular libremente con cero accidentes en las ciudades. Los CEO de las empresas de tecnología de la información (TI) enfatizan el uso de la inteligencia artificial para la actividad intelectual en el planeta, y otra de las predicciones de Kurzweil, relativa a la erradicación de la pobreza en el planeta coincide con el reporte de la Fundación Gates.

Se puede concluir que, sin lugar a duda, la labor de los CEO de las grandes multinacionales, de las empresas creativas e innovadoras, no obstante, su persecución de las utilidades como requisito primordial, está dirigida a llevar a la sociedad hacia cambios insospechados que solamente la dificultad de los seres humanos para acceder a esos cambios puede retrasar.

\section{REFERENCIAS}

Beelitz, A. \& Merkl-Davies, D. M. (2012). Using Discourse to Restore Organizational Legitimacy: CEO Speak after an Incident in a German Nuclear Plant. Journal of Business Ethics, 108, 101-120. DOI: https:// doi.org/10.1007/s10551-011-1065-9

Carollo, L. \& Guerci, M. J. (2018). Activists in a Suit': Paradoxes and Metaphors in Sustainability Managers' Identity Work. Journal of business ethics, 148(2), 249-268. DOI: https://doi.org/10.1007/s10551-017$3582-7$
Craig, R. \& Amernic, J. (2018). ¿Are there language markers of hubris in CEOs letters to shareholders? Journal of business ethics, 149(4), 973-986. DOI: https:// doi.org/10.1007/s10551-016-3100-3

Dewan, M. H. (2017). Discourse Analysis: An Emerging Trend in Corporate Narrative Research. Middle East Journal of business, 12(4), 3-9. Recuperado de file://C:/Users/gutie/Downloads/MEJBOctober2017_split_1.pdf

Diamandis, P. (2018). Ray Kurwweil's Mind-Boggling Predictions for the Next 25 Years. Recuperado de https:// medium.com/@singularity_41680/ray-kurzweilsmind-boggling-predictions-for-the-next-25-yearsce3c9163588b

Fink, L. (2019). Carta anual de 2019 de Larry Fink a los directores propósito y rentabilidad. Recuperado de https://www.blackrock.com/mx/2019-larry-finkcarta-anual

Fortune 500: Revista de Negocios. (2019). Obtenido de http://fortune.com/fortune global500/ 2019.

Glaser, B. \& Strauss, A. (2006). The Discovery of Grounded Theory: Strategies for Qualitative Research. New Brunswick USA: Aldine Transaction.

Hernández-Sampieri, R., Fernández, C. \& Bautista, P. (2010). Metodología de la investigación (5.a ed.). Ciudad de México: Mc Graw Hill. Interamericana Editores S.A.

Herzberg, F. (1959). The motivation to work. New York: John Wiley and Sons.

Hodge, B. (2010). Oedipus and the CEO: Ambiguities of Change in Myth, Discourse and Practice. Culture 
and Organization, 16(1), 23-35. DOI: https://doi. org/10.1080/14759550903558045

Hyland, K. (1998). Exploring Corporate Rethoric: Metadiscourse in the CEO's Letter. The journal of business communication, 35(2), 224-245. Doi: https://doi. org/10.1177/002194369803500203

Jaques, E. (1951). The Changing Culture of a Factory. Londres: Tavistock Institute.

Jaques, E. (2000). A General Theory of Bureaucracy. Portsmouth, NH, USA: Heinemann Educational Books, Inc.

Jaques, E. (2006). Requisite Organization. Baltimore, Estados Unidos: Cason Hall \& Co. Publishers.

Jaques, E. \& Cason, K. (2013). Human Capability. Falls Church, VA, Estados Unidos: Cason Hall \& Co. Publishers.

Kakabadse, A. (2006). Chairman and Chief Executive Officer CEO: That Sacred and Secret Relationship. Journal of management development, 25(2), 134-150. DOI: https://doi.org/10.1108/02621710610645126

Kariskova, D., Green, S. \& Le Breton, J. (2013). Destructive leadership: a theoretical review, integration and future research agenda. Journal of management, 39, 1308. DOI: https://doi. org/10.1177/0149206312471388

Kurzweil, R. (1999). La era de las máquinas espirituales. Barcelona, España: Planeta.

Mc Allum, M. (2014). Why we are at start of an industrial revolution. Recuperado de https://federation.edu. au/_data/assets/pdf_file/0008/238382/The-ThirdIndustrial-Revolution.pdf
Mintzberg, H. (2017). Henry Mintzberg, Co-Founder of Coaching Ourselves, on Managemen. Recuperado de https://www.youtube.com/watch?v=B1YNhr-xeL0

Mintzberg, H. (Abril de 2018). Strategy and Profitability. Entrevista videograbada. Recuperado https://www. youtube.com/watch? $v=$ Wi78IXfurgA

Morton, J., Stacy, P. \& Mohn, M. (2018). Building and Maintaining Strategic Agility. An Agenda and Framework for IT Leaders. California management review, 61(1), 94-113. DOI: https://doi. org/10.1177/0008125618790245

Oppenheimer, A. (2018). jSálvese quien pueda! El futuro del trabajo en la era de la automatización. Ciudad de México: Penguin Random House Grupo Editorial.

Ou, A., Tsui, A., Kinicki, A., Waldman, D., Xiao, Z. \& Jiwen Song, L. (2014). Humble Chief Executive Officers' Connections to Top Management Team Integration and Middle Managers' Responses. Administrative science quarterly, 59(1), 34-72. DOI: https://doi.org/10.1177/0001839213520131

Paroutis, S., McKeown, M. \& Collinson, S. (2013). Bulding Castles from Sand: Unlocking CEO Mythopoetical Behavior in Hewlett Packard from 1978 to 2005. Business History, 55(7), 1200-1227. DOI: https://doi.org/10.1080/00076791.2013. 838038

Patelli, L. \& Pedrini, M. (2014). Is the optimism in CEO's letters to shareholders sincere? Impression management versus communicative action during the economic crisis. Journal of business ethics, 124, 1934. DOI: https://doi.org/10.1007/s10551-013-1855-3 
Pentland, A. (2014). Social physics: how good ideas spread. The lessons from a new science. New York, NY: Penguin Press.

Pérez-Porto, J. \& Gardey, A. (2016). Definición de singularidad. Recuperado de https://definicion.de/ singularidad/

O’Reilly, C. \& Chatman, J. (1994). Working Smarter and Harder: A Longitudinal Study on Managerial Success. Administrative science quarterly, 39(4), 603-627. DOI: https://www.jstor.org/stable/2393773

O'Reilly, C., Doer, B. \& Chatman, J. (2017). "See You in Court": How CEO narcissism increases firms' vulnerability to lawsuits. The leadership quarterly, 29(3), 365-378. DOI: https://doi.org/10.1016/j.leaqua.2017.08.001

Reilly, C., Roer, B., Caldwell, D. \& Chatman, J. (2013). Narcissistic CEOs and Executive Compensation. The leadership quarterly, 25(2), 218-231. DOI: https:// doi.org/10.1016/j.leaqua.2013.08.002

Rifkin, J. (2013). México frente a la tercera revolución industrial del siglo XXI. Biblioteca Jurídica Virtual del Instituto de Investigaciones Jurídicas de la UNAM. Recuperado de http://biblio.juridicas.unam.mx

Rifkin, J. (2016). Cinco días. El País. Economía. Recuperado de https://cincodias.elpais.com/cincodias/2014/09/19/economia/1411142749_435160. html

Rousseau, D. (1989). Psychological and Implied Contracts in Organizations. Employee responsibilities and rights, 2(1), 121-139. DOI https://doi.org/10.1007/ BF01384942
Sagan, C. (1980). Cosmos. Barcelona: Planeta.

Toffler, A. (1970). El shock del futuro. Barcelona: Plaza y Janés S.A. Editores.

Toffler, A. (1979). La tercera ola. Barcelona: Plaza y Janés Editores. 